\title{
Retrograde Coronary Chronic Total Occlusion Intervention
}

\author{
Debabrata Dash* \\ S. L Raheja (A Fortis Associate) Hospital, Raheja Rugnalaya Marg, Mahim (West), Mumbai, 400016, \\ India
}

\begin{abstract}
Coronary chronic total occlusion (CTO) is a frequent finding in patients with coronary artery disease. It remains one of the most challenging subsets, accounting for $10-20 \%$ of all percutaneous coronary interventions (PCI). Although remarkable progress in PCI has been made, it is reasonable to state that successful recanalization of CTO represents the "last frontier" of PCI. PCI of CTOs has been limited historically by technical success rates of $50-70 \%$. The introduction of enhanced guidewires, microcatheter, channel dilatator with increasing operator experience, and innovative techniques such as the retrograde approach have raised hopes for better outcomes. This article goes into depth into various strategies of retrograde approach in CTO.
\end{abstract}

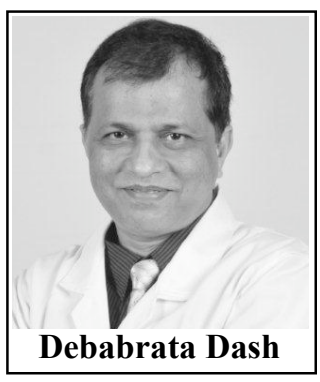

Keywords: Chronic total occlusion, percutaneous coronary intervention, retrograde approach.

\section{INTRODUCTION}

It has been demonstrated that successful recanalization of CTO improves angina status, increases exercise capacity, and reduces mortality $[1,2]$ and the need for subsequent coronary artery bypass surgery [3]. Despite remarkable progress over the last decades, successful recanalization of CTOs represents the "final frontier" of PCI. PCI of this subset of lesions is still technically challenging although new devices and a wide range of guidewires have been developed $[4,5]$. The technical success rate for CTO via the antegrade approach remains suboptimal, in the range of $65 \%-70 \%$ worldwide $[1,6]$. However, retrograde approach via collateral channel (CC) overcomes the limitations of the conventional antegrade approach. When it becomes more widely adopted it is expected to be a breakthrough to potentially improve the success rate of complex CTO [7-9]. Despite this, adoption of this technique is limited. Retrograde procedure is used in only $15 \%$ of CTO PCI as suggested by the EuroCTO registry [10].

\section{INDICATIONS FOR RETROGRADE APPROACH}

Retrograde access via donor collateral (bypass graft) was first performed in 1990 [11]. A higher success rate upto $95 \%$ could be achieved from the combination of antegrade and retrograde approach. Angiographic features of blunt occlusion with a large side branch, bridging collaterals, calcification, and long CTO are no longer considered negative predictors of success while using a retrograde approach [12]. Possible indications of this new approach could be subsets of previous antegrade failures. It could be the primary procedure in many situations like ostial occlusions, long occlusions, heavy calcification, occlusions with ambiguous

*Address correspondence to this author at the S. L Raheja (A Fortis Associate) Hospital, Raheja Rugnalaya Marg, Mahim (West), Mumbai, 400016, India; Tel: +91-22-6652 9666; Fax: +91-22-2444-2486; E-mail: dr_dash2003@yahoo.com proximal cap, and occlusions with a diffusely diseased distal vessel. Retrograde penetration of the distal CTO cap may be easier because it is tapered and softer as compared to the proximal cap; also, it is not reliant upon precise visualization of CTO origin, and is exposed to higher pressure, where the proximal cap is blunt or less benign.

\section{ANGIOGRAPHIC/ASSESSMENT AND SELECTION COLLATERAL CHANNEL ANALYSIS}

The first key to success of the retrograde approach is to study the diagnostic images carefully and select a best CC. Optimal angiographic planning requires dual injections from both left and right coronary ostia with complete filling of the distal collateral bed from all feeding sources. Lower magnification and avoiding panning considerably helps in evaluating CCs. The distal vessel size, nature of the proximal and distal cap, side branches, tortuosity, and calcification are other critical items of information that are gained from the angiogram.

The three types of CCs are bypass graft, epicardial channels, and septal channels.

In severely degenerated saphenous vein graft cases, it is necessary to recanalize CTO of the native coronary artery to improve long-term outcome. Despite the poor suitability of graft for PCI, it can be used as a channel for wire passage. An arterial graft could also be used for the retrograde conduit.

Septal CCs are the safest and are preferable whenever possible. Compared with the epicardial, the septal CC is a shorter route to the recipient vessel and is less tortuous. It can also be dilated using a small balloon $(1.25-1.5 \mathrm{~mm})$ with very low pressure (up to $2 \mathrm{~atm}$ ) to navigate the catheter through it without major adverse clinical events [13]. At present, the Corsair catheter (Asahi Intec, Japan) circumvents septal CCs dilatation with a small balloon. Excessive septal tortuosity is a severe limitation to wire advancement whereas size is less so. Straight faintly visible or even invisible septal 
CCs could be crossed by "surfing" with the wire [14]. The angle of entry, angle of donor vessel and at the base of the heart, if acute, could be a significant limitation to both wire and microcatheter passage. These CCs are best evaluated in a combination of right anterior oblique (RAO) or anteroposterior cranial (AP) and RAO projections.

As opposed to septal CCs, the main assessment criteria for epicardial CCs is a size and not the extent of tortuosity. Challenging anatomy, such as a corkscrew or highly tortuous configuration demonstrated by epicardial CCs, is not a contraindication, especially in the Corsair era. In general, larger epicardial CCs are safer and more likely to permit a balloon or microcatheter. There is some risk of perforation during wire manipulation which could be controlled by simple ballooning at the proximal site. Dilatation of this CC should not be done as it leads to vessel rupture and cardiac tamponade. However, the chance for tamponade is lower during wiring of the epicardial CC in a patient with bypass graft as there is no pericardial space. When this channel is the only donor artery to a viable CTO region, wire manipulation leads to distant ischemia.

\section{SYSTEM SETUP}

Two 6-8 F guiding catheters are usually engaged in the right and left coronary arteries via radial or femoral arteries. Use of bi-femoral or radial route is a matter of operator preference and experience with knowledge of risks/benefit of both. For retrograde limb, use of short $(90 \mathrm{~cm})$ guiding catheters (especially when using epicardial CCs) allowing the externalization of the wire, is recommended. A regular 100 $\mathrm{cm}$ guide catheter can be manually shortened and connected to a $1 \mathrm{~F}$ smaller introducer sheath [15]. Removing $10 \mathrm{~cm}$ is usually sufficient. Because of reversibility, heparin is the anticoagulation of choice. An activated clotting time (ACT) of 300 to 350 seconds should be targeted. Bivaluridin or glycoprotein IIb/IIIa antagonists are not recommended because of concerns in case of perforation.

\section{COLLATERAL CROSSING AND GUIDEWIRE HAN- DLING}

Successful collateral crossing depends on CC selection, wire tip curve, and wire handling.

The best CC would be clearly visible, less tortuous collaterals by super-selective injection, exemplified by Dr. Werner's CC grade 1 or 2 (Fig. 1A and B) (CCs are graded as follows: $\mathrm{CC} 0$, no continuous connection, $\mathrm{CC} 1$, continuous thread-like connection; and $\mathrm{CC} 2$, continuous, small sidebranch-like connection) [7]. Acute angulation, branching, cork-screw morphology, and calcification are major hurdles for wire crossing [12]. It would be wise to avoid severe corkscrew-like collaterals, which are characterized by an amplitude to diameter ratio of less than 2. Suitable for CC crossing, the best spring coil wires are Sion and Sion blue (Asahi Intec, Japan); polymer jacket wires are Fielder FC, XT, XTR (Asahi Intec, Japan), Pilot 50, and Whisper (Abbott vascular, USA). Fielder FC would be of choice in CC 1 or 2. Fielder XT, XTR or Sion (Asahi Intec, Japan) wire would be recommended in less visible, minor tortuosity on CC 0 or 1 . Sion blue (Asahi Intec, Japan) can help to track very tiny or invisible CCs, or CCs with threedimensional tortuosity.

An extremely small tip $(<1 \mathrm{~mm})$ curve $\left(45^{\circ}\right)$ is recommended for $\mathrm{CC}$ navigation. After a wire access to the selected CC, the microcatheter [Corsair (Asahi Intec, Japan), Fine cross (Terumo, Japan)] is placed at the start of the CC. Although choice of microcatheter depends on operator preference, currently Corsair is the microcatheter of choice. After the position has been secured, the workhorse wire is exchanged with an appropriate polymer jacket (tapered or nontapered) or spring coil wire to cross the CC.

The Corsair is an over the wire (OTW) hybrid catheter composed of 8 thin wires wound with 2 large ones. It has a soft tapered tip with tungsten braiding and a hydrophilic shaft which serves as a CC dilator while providing exceptional CC tracking and crossing as well as retrograde wire control. Gentle rotation of this catheter is required to advance or pull it by either clockwise or counterclockwise rotation. It is performed by 5-10 alternating clockwise and counterclockwise rotations while providing forward tension. Rotation should be limited to 10 times in one direction. In case of failure to cross or blockage of the Corsair, it should be exchanged for a low-profile microcatheter (Finecross, Terumo, Japan) or an OTW balloon (Ryujin $1.25 \mathrm{~mm} \times 10 \mathrm{~mm}$ Terumo, Japan; Lacross $1.30 \mathrm{~mm}$ x $10 \mathrm{~mm}$, Goodman, Japan or Maverick $1.50 \mathrm{~mm} \times 15 \mathrm{~mm}$, Boston Scientific, USA) with a longer shaft $(>145 \mathrm{~cm})$ by using extension guidewire or the counter flush method. From the very beginning, the Finecross should be the microcatheter of choice for a very tortuous epicardial channel. The author prefers the newly developed Finecross GT (Asahi Intec, Japan) for locating channels with small bends.
A)

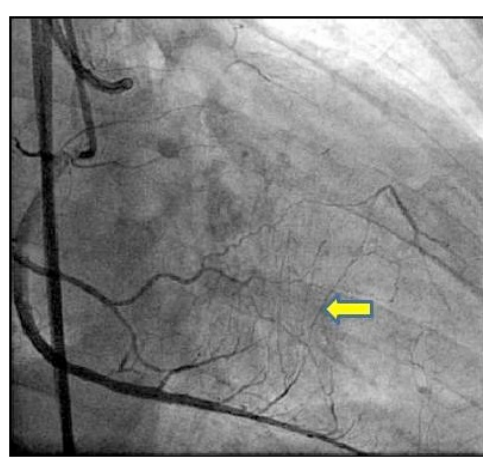

B)

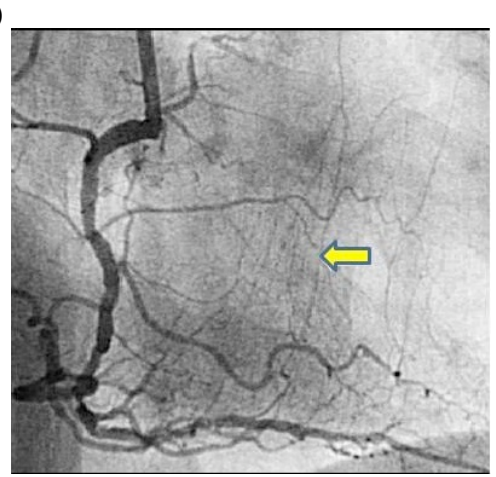

Fig. (1). A: Werner's collateral channel grade 1. B: Werner's collateral channel grade 2. 
Once the wire is positioned at the tip of the corsair catheter, it is gently advanced with rotation along the path of least resistance (surfing) in case of septal CCs and should halt once a series of ventricular beats has developed or migration to the side branch is noted. The wire is quickly redirected to find an alternative CCs in case of resistance. Forceful advancement of the wire could lead to CC rupture, vessel perforation, and septal hematoma which might be fatal. In doubtful wire position there is selective injection (advancement of microcatheter with pulling the back of the wire) of the contrast (as little as $2 \mathrm{ml}$ ); if a wash out of the contrast is revealed, the $\mathrm{CC}$ is connected to the other ventricle. Wire advancement in to the left or right ventricle is benign as long as the microcatheter is not advanced. When the contrast stays in the channel, it infers focal perforation. Selective injection is the last resort after several surfing attempts have failed, as it increases the risk of septal rupture and hematoma. During septal surfing, the wrong path is also assessed by wire buckling. On occasion, there are sudden rapid and large deviations in wire-tip movement indicating entry into a cardiac chamber. Septal hematoma, manifesting as severe chest pain, may lead to a fatal event if not treated adequately, such as by coil embolization of septal CC or creation of a fistula connecting to the ventricle [14].

In case of epicardial CC, surfing is not advisable. Divergence from the observed path may lead to perforation which is more problematic than septal rupture.

\section{CONNECTING THE ANTEGRADE AND RETRO- GRADE CHANNEL}

Retrograde penetration is attempted with a guidewire such as a Fielder XT (Asahi Intec, Japan) from the distal cap of a CTO lesion with the support of a microcatheter. If it fails, it should be exchanged for an Ultimate Bro 3, Miracle 3, 6 and 12 (Asahi Intec, Japan) and is advanced in a retrograde manner to the most proximal part possible. The recently introduced Gaia 2(Asahi Intec, Japan) is effective for retrograde tracking of true lumen. If the distal cap is very hard and calcified, Conquest Pro or Conquest Pro 12 (Asahi
Intec, Japan) should be used. While keeping the retrograde approach in place, an antegrade approach is also commenced. After successful penetration of the tip of the retrograde guidewire into the CTO lesion, one or a combination of the following different strategies is used $[9,16]$.

\section{RETROGRADE WIRE CROSSING TECHNIQUE}

When a wire crosses the entire occlusion in a retrograde manner, it is called "retrograde wire crossing". After deeply negotiating the retrograde wire into the aorta or the antegrade guide, it can be anchored by inflating a small balloon (2-2.5 $\mathrm{mm}$ ) within the antegrade guide catheter to facilitate crossing of the occlusion with the Corsair (Figs. 2, 3). If this fails, the Corsair is exchanged for a 1.25 or $1.5 \mathrm{~mm}$ OTW balloon, which usually has a smaller crossing profile than the Corsair to perform the retrograde balloon dilatation in the occlusion so that any subsequent procedure can be accomplished in the antegrade manner. This is the simplest way of all the retrograde techniques and can be achieved in $30 \%$ of cases, but it is the view of the author that the success rate could be $50 \%$ with appropriate wire escalation. When direct retrograde wire crossing fails, other techniques to connect the antegrade and retrograde channels are the kissing wire, controlled antegrade and retrograde subintimal tracking (CART) technique, Reverse CART, and knuckle wire technique (KWT).

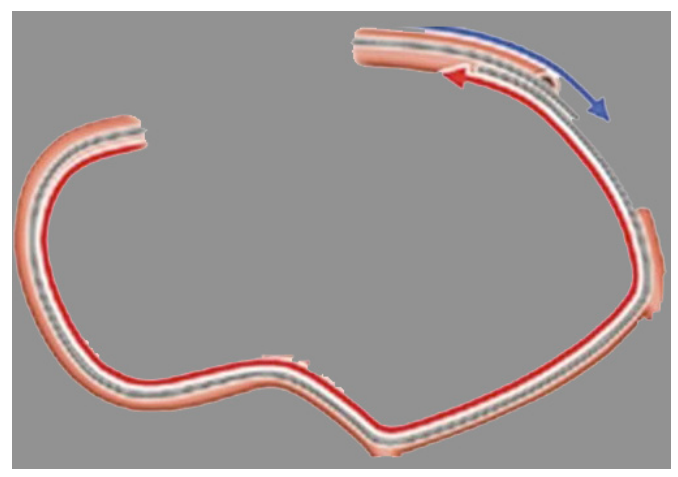

Fig. (2). Retrograde wire crossing.

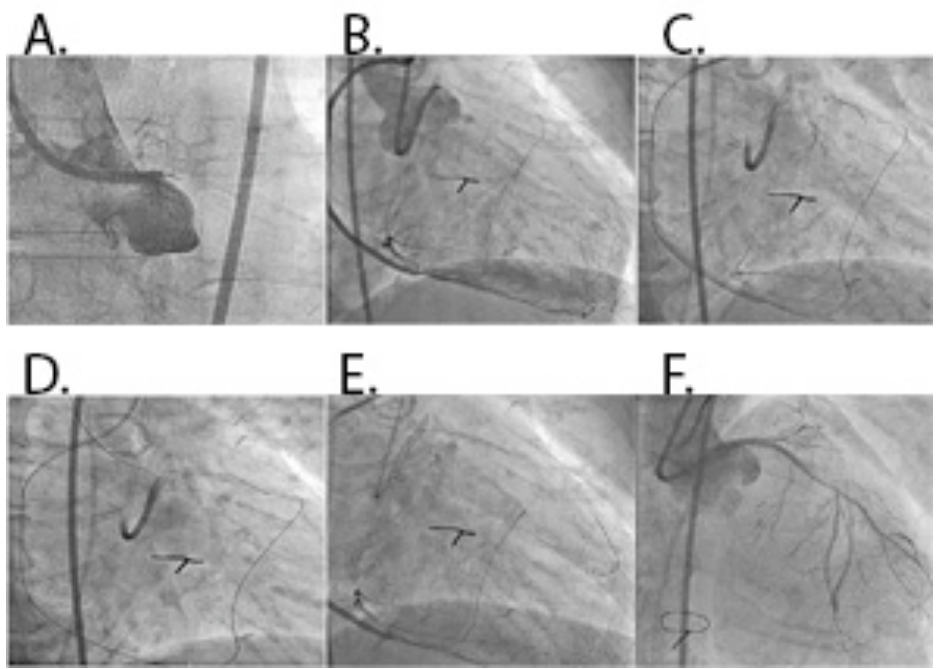

Fig. (3). A: CTO of left main coronary artery (LMCA). B: Werner's collateral channel grade 2 to saphenous vein graft-RCA to LAD. C: Retrograde guidewire in LAD with microcatheter. D: Retrograde wire crossing. E: Antegrade wire navigation into LAD. F: Final result after implantation of DES in LMCA to LAD. 


\section{KISSING WIRE TECHNIQUE}

The retrograde wire is advanced to the proximal part within the CTO lesion. If the tip of this guidewire approaches the proximal cap of the CTO with its tip in alignment with the antegrade guidewire, both the antegrade and retrograde guidewires (Figs. 4, 5) meet (kiss) [17] (Fig. 1). However, it is difficult to align both wires inside the occluded true channel as there are many diseased layers inside the occlusion.

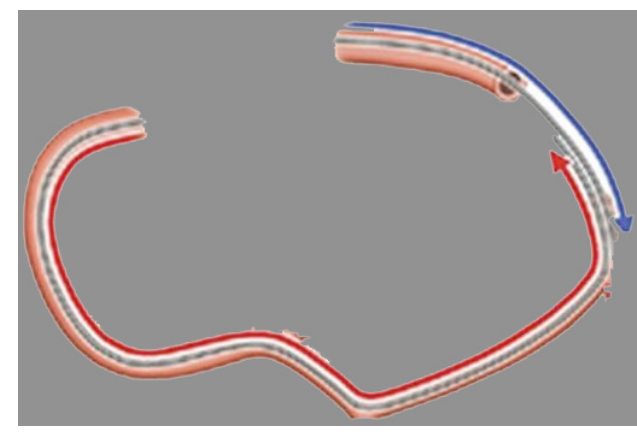

Fig. (4). Kissing wire cross.

\section{THE CART TECHNIQUE}

The CART technique consists of antegrade wiring through the CTO where retrograde balloon creates a local subintimal dissection for facilitating wire crossing to the distal true lumen [18]. At first, a wire is advanced antegradely, trying to cross the CTO. If the wire has entered the subintimal space, it is left in this position. A second wire is advanced in a retrograde manner through the $\mathrm{CC}$ under the support of a microcatheter. This wire is positioned at the distal end of the CTO, then penetrated retrogradely from the distal true lumen into the $\mathrm{CTO}$, and finally into the subintimal space at the CTO site. A small balloon $(1.5-2 \mathrm{~mm})$ is advanced over the retrograde wire into the subintimal space; it is then dilated in the same space and also on the course from this subintimal space to the proximal cap of the CTO. The deflated balloon should be left in place to keep this subintimal space open. This enables two dissections (created by antegrade wire and the retrograde balloon) to lie in the subintimal space at the CTO site. Thereafter, the antegrade wire is advanced further along the deflated retrograde balloon that lies from the subintimal space to the distal true lumen (Fig. 6). Then ballooning and stent implantation can be performed over the antegrade wire [19]. The advantage of this technique is the minimization of just subintimal tracking through the length of the CTO lesion. The limitation is that, it is not always possible to negotiate a retrograde balloon inside the occlusion, particularly in complex CTO lesions. Subintimal space extending to the proximal true lumen of the CTO may cause a fatal event when the CTO is located in the proximal part of the left coronary system (e.g. dissection of the left main) [20]. Other limitations are empiric estimation of retrograde balloon size and unpredictable procedure time. It is advisable to use the closest sized balloon inside the CTO to create sufficient wire re-entry space. With introduction of the Corsair microcatheter the CART technique is rarely employed except in some cases of ostial occlusions, heavily calcified occlusions, and when the microcatheter is unable to enter the occlusion.

\section{REVERSE CART TECHNIQUE}

The reverse CART technique consists of dilatating the CTO lesion with the balloon through the antegrade wire followed by retrograde wire being advanced into the space dilated with the antegrade balloon [21]. A wire is negotiated in a retrograde manner into the distal cap of the CTO and another is advanced antegradely in the proximal cap of the CTO. The retrograde wire is advanced into the subintimal space into the CTO lesion. The subintimal space created by antegrade wire is enlarged by inflating a balloon to create a plaque dissection and modification of the lesion which is target space for retrograde wire penetration into the proximal

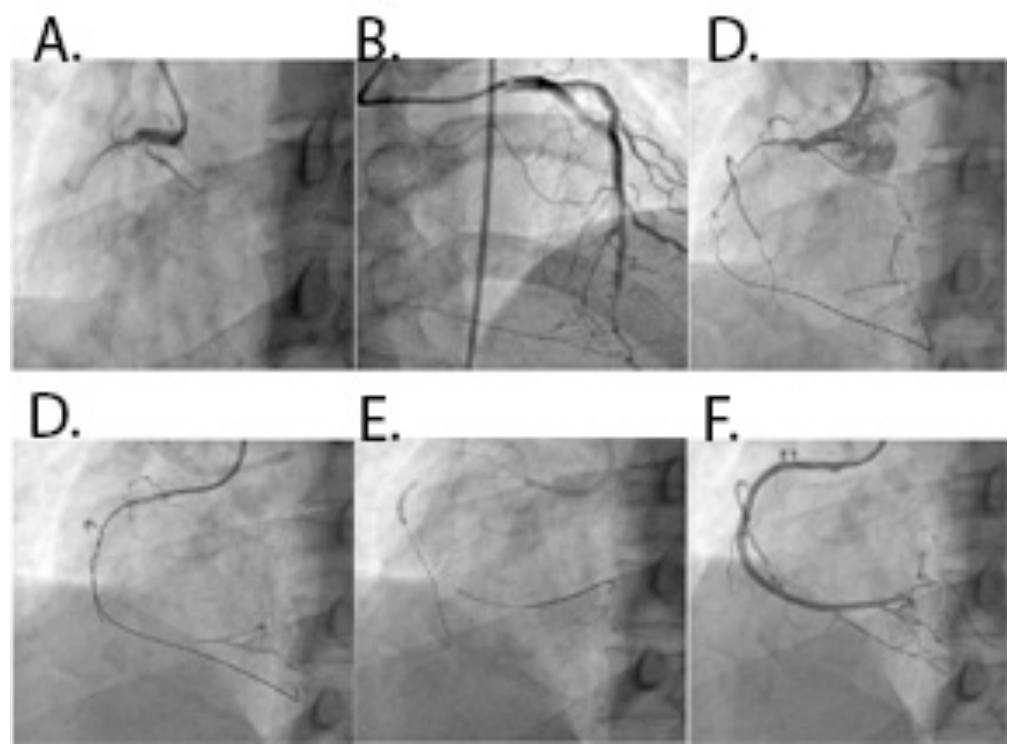

Fig. (5). A: CTO of proximal RCA. B: Werner's collateral channel grade 2 to RCA from LAD. C: Retrograde guidewire in RCA with microcatheter. D: Kissing of antegrade and retrograde guidewires. E: Advancement of DES with balloon anchor in side branch. F: Final result after implantation 2 overlapping DES. 

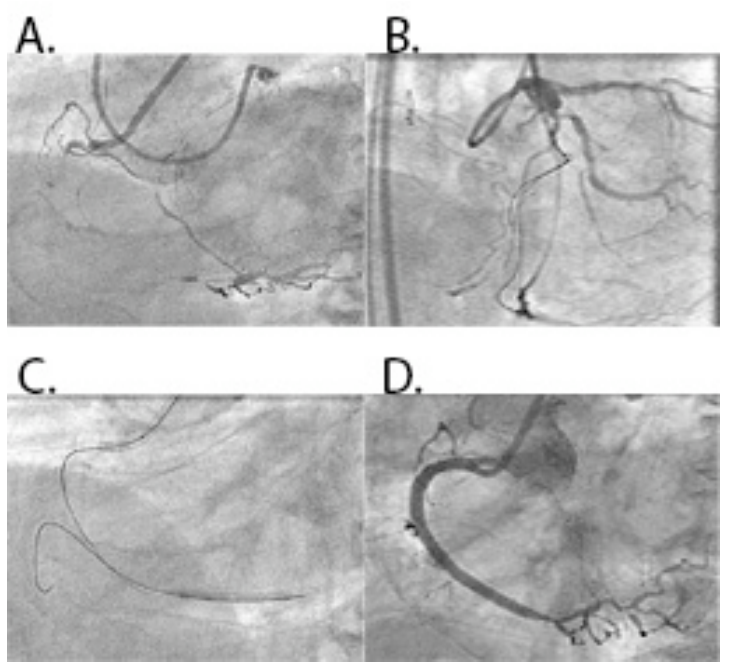

Fig. (6). A: CTO of proximal RCA with good epicardial collateral. B: Retrograde guidewire in epicardial collateral with support of microcatheter. C: Dilatation of subintimal space with a small balloon retrogradely followed by antegrade advancement of wire along the deflated balloon. D: Final result after two overlapping DES in RCA.

true lumen. Next, the retrograde wire is externalized through the guide and is used for subsequent antegrade angioplasty (Figs. 7, 8). The tip of retrograde microcatheter should be in the mid portion of the antegrade balloon. Before balloon inflation, the balloon and retrograde microcatheter may lie 4 to $5 \mathrm{~mm}$ apart on fluoroscopy, yet they are both in subintimal space. With balloon inflation, the two subintimal spaces become one space. There should be no space between the balloon and the retrograde microcatheter. If a gap exists, there remains tissue between the two and the common space does not exist. In this scenario, either a higher inflation pressure is required or a larger diameter balloon needs to be used. On deflation of the balloon, the retrograde support catheter can be seen "drooping" into the common space created indicating a continuous pathway from distal true lumen, through the subintimal space, and into the proximal true lumen. This technique is predictable and reproducible as compared to classical CART. Undersizing of the antegrade balloon makes the creation of a common subintimal space much more difficult. The subintimal space may either compress or collapse after antegrade balloon inflation or deflation, making the true lumen wiring more difficult even with continuous subintimal connection. The "Stent Reverse CART" technique entails deployment of a stent within the antegrade dissected plane to create an open target for retrograde crossing [14]. An alternative method is to use a Guideliner mother-in-child catheter (Vascular solutions, Minneapolis, MN, USA) into the space to help connect the retrograde wire to the antegrade guide (Mother-in-child Reverse CART) [14]. Unlike a stent, a catheter may be removed or repositioned if the connection between the antegrade and the retrograde true lumen fails.

Recoil of subintimal channel may occur even after successfully connecting the channel between the antegrade and retrograde space. Moreover, blind medial disruption with the antegrade balloon potentially causes bidirectional expansion of the subintimal dissection making retrograde wire crossing difficult. The intravascular ultrasound (IVUS) estimates optimal antegrade balloon size that avoids risk of perforation. A smaller size balloon should be used in presence of calcified plaque. If IVUS indicates the recoil of a connecting channel, redilatation with a bigger balloon should be performed. IVUS also directly visualizes the position of retrograde wire in the subintima [21]. The retrograde wire could be navigated into proximal true lumen under IVUS (IVUSguided reverse CART) [22]. An important precaution is to avoid contrast injections after creating antegrade subintimal dissection until the last stent is deployed to prevent pressurized contrast injection into the dilated segment causing a spiral dissection or hematoma.

\section{THE KWT TECHNIQUE}

The KWT technique is employed in case of failure of retrograde wire escalation or when the CTO is quite long with vessel ambiguity. This entails creating a small loop with retrograde polymer jacketed wire Fielder FC, XT (Asahi Intec, Japan) or Pilot 200 (Abbott Vascular, USA), which is advanced inside the occlusion and creates subintimal space so that antegrade wire in the subintima can be led inside this space. The antegrade KWT can be combined with retrograde KWT in complex CTO lesions. The limitations of this technique are such that a longitudinal dissected plane cannot be controlled and the cross-sectional dissected wire is not wide enough to lead the antegrade wire [23]. Knuckle wire should be pushed, but never rotated for fear of wire knotting.

\section{WIRE TRAPPING TECHNIQUE}

After retrograde wire crossing, the antegrade wire can be trapped in the distal lumen by an inflated balloon to create an extra back up force for balloon crossing or stent delivery. In retrograde wire crossing or reverse CART technique, retrograde wire can be trapped in the proximal lumen or in the guide catheter for easy passage of retrograde balloon inside the occlusion.

After the retrograde wire enters the ascending aorta, a gooseneck microsnare (Amplatz, USA) is inserted and snares the retrograde wire tip from the aorta into the antegrade guiding catheter. The microsnare is pulled back slowly and carefully until retrograde wire is pulled out of the antegrade 


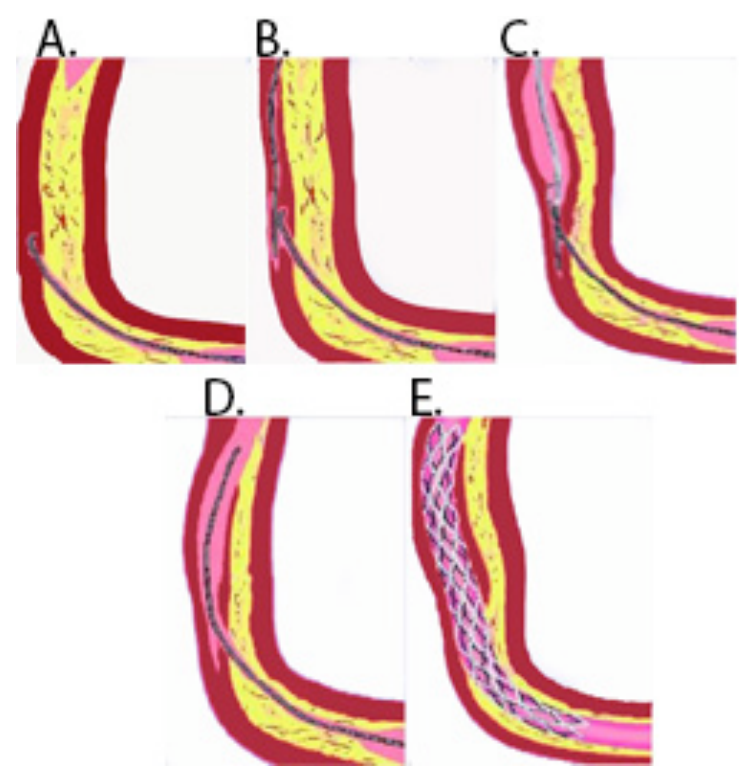

Fig. (7). A: Retrograde guide wire in subintima of distal cap with microcatheter. B: Antegrade guidewire insubintimal space. C: Connection of both antegrade and retrograde subintimal space after balloon dilatation of subintimal space antegradely. D: Retrograde guidewire advancement into true lumen with microcatheter. E: Retrogarde guidewire externalization followed by stenting anterogradely.

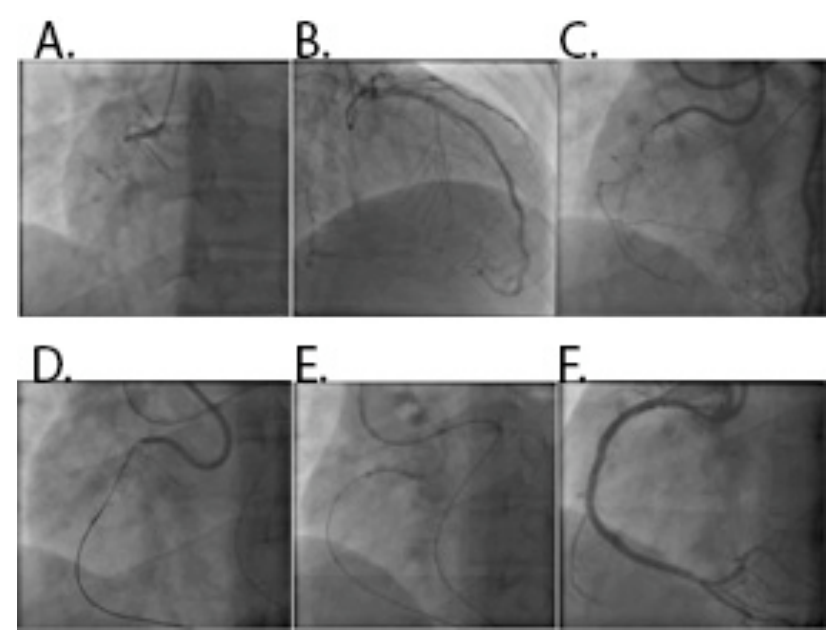

Fig. (8). A. CTO of proximal RCA. B: Werner's collateral channel grade 2 collateral to PDA from LAD. C: Failure of antegrade approach. D: Retrograde guidewire navigated through septal collateral channel with channel dilataor (Corsair) support. E: Penetration of retrograde guidewire into true lumen of RCA after balloon dilatation of subintimal space antegradely. F: Final result after.

guiding catheter. The balloon and stent can be delivered over retrograde wire to recanalize the occlusion [24].

\section{REVERSE WIRE TRAPPING TECHNIQUE}

After crossing the CTO lesion, entering the ascending aorta, and being snared by the antegrade microsnare, the retrograde wire is drawn back, dragging the microsnare tip to advance through the occlusion simultaneously. After the microsnare and microcatheter have crossed the lesion antegradely, the retrograde wire is released. Afterwards the microsnare is drawn back and exchanged for an antegrade wire followed by balloon dilatation and stent implantation [25].

\section{THE RENDEZVOUS (BRIDGE) TECHNIQUE}

In this technique, antegrade and retrograde microcatheters are aligned in a guide catheter at the level of the ascending aorta, after which an antegrade wire is pulled into a ret- rograde microcatheter. The antegrade wire is advanced further down beyond the CTO site through the retrograde microcatheter [26]. This bridge technique could be used in case of failure of advancement of antegrade wire, whereas retrograde wire can be negotiated using a microcatheter. This can also be applied in cases of large dissection and uncertainty in the movement of wire, or in cases where CCs are small and not ideal for large retrograde balloon dilatation. This can replace wire externalization by $300-\mathrm{cm}$ wire.

\section{DOUBLE BALLOON INFLATION}

Two overlapping balloons inserted in the retrograde and antegrade subintimal spaces are inflated simultaneously to join the subintimal spaces together with confluence in Reverse CART technique. This allows the retrograde wire to pass easily through this newly created confluent subintimal space and then finally into true lumen [27]. 


\section{EXTERNALIZATION}

The crossing wire is exchanged for an externalization wire after a microcatheter is delivered into the antegrade guide. The externalization of the retrograde wire must be carried out with utmost care. The retrograde guide catheter may be deep throated; this needs to be avoided to prevent ostial damage. While advancing the stiffer shaft of the retrograde wire into the $\mathrm{CC}$, the collateral needs to be protected by the presence of the microcatheter.

Several workhorse wires come in $\geq 300 \mathrm{~cm}$ (300-cm Pilot 200 wire, Abbott Vascular, USA; $325 \mathrm{~cm}$ Rota floppy, Boston Scientific, USA; $335 \mathrm{~cm}$ Viper, CSI, St Paul, Minnesota; $300 \mathrm{~cm}$ RG3, Fielder FC, Asahi Intec, Japan) for externalization. The Viper wire is extraordinarily stiff, and is often difficult to pass through very tortuous CCs. This has been overcome by flushing the channel dilator with Rotaglide solution (Boston Scientific, USA) before the externalization wire is inserted. The shaft of the Rota floppy wire is only 0.009 " in diameter, and is easily kinkable. It should be used as a last resort if no other long wires are available. RG3 wire has emerged as an ideal guidewire. However, the shaft is not as stiff as a standard coronary wire and therefore it gives less support to balloons and stents after externalization.

Despite coaxial antegrade guide, retrograde passage of wire into guide fails in conditions such as in aorto-ostial lesions or extremely tortuous vessels, or whenever there is poor retrograde wire control. Difficulty in wiring the antegrade guide can be overcome by snaring. The 3-snare system, referred to as tulip snare (EN Snare; Merit Medical Systems, South Jordan, USA), is the most useful snaring system for externalization of wire during retrograde procedure. The larger the snare the better it is. An $18 \times 30 \mathrm{~mm}$ EN Snare, which is 6 French compatible, is preferred [28, 29].

\section{FINAL TREATMENT}

Once the externalized wire approaches the antegrade guiding hub, the operator needs to detach the copilot, place a finger over the hub of antegrade guide, and wait for retrograde wire to tap it. Retrograde wire is pushed 5 to $7 \mathrm{~cm}$ out of the guide, once the tap is felt. Then a wire introducer is placed into antegrade copilot and the externalized soft tip of the wire is threaded. Introducer and copilot over the wire are slided and reconnected without flushing. Flushing after reverse CART can result in hydraulic dissection. Ballooning and stenting is performed after 20 to $30 \mathrm{~cm}$ wire is externalized. Retrograde injection is used for a distal landing zone for stents. Externalized wire in a tortuous artery should always be protected with a catheter. The stiff part of the wire should be under cover of a channel dilator or channelcrossing catheter. The end of the externalized wire should never be pulled unless the $\mathrm{CC}$ is protected. However, pushing is always permitted but still it needs to be done with protection of the CCs [14, 30].

\section{REMOVAL OF THE EXTERNALIZED WIRE AND CORSAIR CHANNEL DILATOR}

To remove the wire, the channel dilator should be readvanced into the antegrade guide. It must protect the $\mathrm{CC}$ until the soft wire tip is back in the CC. Both antegrade and retro- grade guiding catheters are disengaged from the coronary ostium and pulled back 3 to $4 \mathrm{~cm}$ into the aorta to avoid ostial dissection because of externalized wire retraction. After establishing guiding catheter control, the externalized wire is removed and gradually synchronized with the heart beat taking care not to kink it. Then the channel dilator is removed with clockwise rotation. However, some operators advocate leaving some part of the wire within the channel dilator to retract it so that $\mathrm{CC}$ can be assessed in the event of its rupture that could otherwise manage this complication rapidly. Once the retrograde system has been removed successfully, a careful angiographic demonstration of the integrity of the CCs, as well as checking for extravasations, needs to be performed via the retrograde catheter.

\section{COMPLICATIONS ASSOCIATED WITH THE PRO- CEDURE}

Specific complications pertaining to retrograde approach are $\mathrm{CC}$ rupture, thrombus formation, and global ischemia. Most CC ruptures are benign and do not require further treatment [11] apart from abandoning that $\mathrm{CC}$ and trying for another. The channel dilator is safer than a balloon and rarely causes CC dissection or perforation even in CC with excessive tortuosity. While advancing the microcatheter, if septal wire shows excessive kinking, the wire has to be withdrawn to prevent septal perforation [29]. If there is small septal perforation to the right or left ventricle, observation can be made. Septal hematoma or cardiac tamponade needs embolic occlusion via microcatheter. Before pulling out the retrograde wire, the donor artery should be visualized with the externalized wire in place. Then a microcatheter from both ends of the wire would allow access to a bleeding point.

Thrombus formation is prevented by saline flush of the guide catheter and by maintenance of ACT $>300$ seconds and checked every 20-30 min. Global ischemia can be minimized by careful manipulation of retrograde wire. Stenting should be done if there is significant stenosis to the $\mathrm{CC}$ of the donor artery. Catheter induced dissection occurs when the retrograde devices are withdrawn. This could be prevented by constant pulling of the guide catheter to disengage from the ostium, while gentle rotation (in either direction) of the channel dilator is performed to withdraw it.

\section{CONCLUSION}

A growing body of evidence demonstrates that successful PCI of CTO translates into clinical benefit. However, because of the perceived procedural complexity, CTO still is the most common reason for discarding PCI. The proven clinical benefit, as well as the improved long-term patency with drug-eluting stents, has resulted in a growing interest among interventionists for treating CTOs. Tremendous improvements in the PCI equipment, and the development of innovative strategies including the retrograde techniques, have allowed for a success rate of up to $95 \%$, even in complex CTO cases. With the application of the soft polymercoated wires, IVUS, and channel dilators like Corsair, more and more would be managed by reverse CART, while conventional CART is still likely to be needed in ostial occlusions, heavily calcified occlusions, and if the microcatheter is unable to enter the occlusions. Collaborative efforts to 
share experience of this retrograde technique and overcome challenges through refinement in technique and technology would be pivotal to further success in CTO PCI.

\section{CONFLICT OF INTEREST}

The author confirms that this article content has no conflict of interest.

\section{ACKNOWLEDGEMENT}

Declared none.

\section{REFERENCES}

[1] Ivanhoe RJ, Weintraub WS, Douglas JS Jr, et al. Percutaneous transluminal coronary angioplasty of chronic total occlusions. Primary success, restenosis, and long-term clinical follow-up. Circulation 1992; 85: 106-15.

[2] Suero JA, Marso SP, Jones PG, et al. Procedural outcomes and long-term survival among patients undergoing percutaneous coronary intervention of a chronic total occlusion in native coronary arteries: A 20-year experience. J Am Coll Cardiol 2001; 38: 409-14.

[3] Warren RJ, Black AJ, Valentine PA, et al. Coronary angioplasty for chronic total occlusion reduces the need for subsequent coronary bypass surgery. Am Heart J 1990; 120: 270-4.

[4] Hoye A, van Domburg RT, Sonnenschein K, et al. Percutaneous coronary intervention for chronic total occlusions: The Thoraxcenter experience 1992-2002. Eur Heart J 2005; 26: 2630-6.

[5] Saito S, Tanaka S, Hiroe Y, et al. Angioplasty for chronic total occlusion by using tapered-tip guidewires. Cathet Cardiovasc Interv 2003; 59: 305-11.

[6] Ruocco Jr NA, Ring ME, Holubkov R, et al. Results of coronary angioplasty of chronic total occlusions (the National Heart, Lung and Registry). Am J Cardiol 1992; 85: 106-15.

[7] Werner GS, Ferrari M, Heinke S, et al. Angiographic assessment of collateral connectios in comparison with invasively determined collateral function in chronic coronary occlusions. Circulation 2003; 107: 1972-7.

[8] Sheiban I, Moretti C, Omede P, et al. The retrograde coronary approach for chronic total occlusions: mid-term results and technical tips and tricks. J Interven Cardiol 2007; 20: 466-73.

[9] Saito S. Different strategies of retrograde approach in coronary angioplasty for chronic total occlusion. Cathet Cardiovasc Interv 2008; 71: 8-19.

[10] Galsssi AR. Tomasello SD, Reifart N, et al. In-hospital outcomes of percutaneous coronary intervention in patients with chronic total occlusion: insights from the ERCTO (European Registry of Chronic Total Occlusion) registry. EuroIntervention 2011; 7: 4729.

[11] Kahn JK, Hartzler GO. Retrograde coronary angioplasty of isolated arterial segments through saphenous vein bypass grafts. Cathet Cardiovasc Diagn 1990; 20: 88-93.

[12] Rathore S, Katoh O, Matsuo H, et al. Retrograde percutaneous recanalization of chronic total occlusion of the coronary arteries: procedural outcomes and predictors of successin contemporary practice. Circ Cardiovasc Interv 2009; 2: 124-32.
[13] Surmely JF, Katoh O, Tsuchikane E, et al. Coronary septal collaterala as an access for the retrograde approach in the percutaneous treatment of coronary chronic total occlusions. Cathet Cardiovasc Interv 2007; 69: 826-32.

[14] Joyal D, Thompson CA, Grantham JA, et al. The retrograde technique technique for recanalization of chronic total occlusion: A step-by-step approach. J Am Coll Cardiol Interv 2012; 5: 1-11.

[15] Wu EB, Chan WW, Yu CM, et al. Retrogarde chronic total occlusion intervention: tips and tricks. Cathet Cardiovasc Interv 2008; 72: 806-14.

[16] Ge J. Current status of percutaneous coronary intervention of chronic total occlusion. Zhejiang Univ-Sci B (Biomed \& Biotechnol) 2012; 13: 589-602.

[17] Niccoli G, Ochiai M, Mazzari M. A complex case of right coronary artery chronic total occlusion treated by a successful multi-step Japanese approach. J Invas Cardiol 2008; 8: E230-E233.

[18] Surmely JF, Tsuchikane E, Katoh O, et al. New concept for CTO recanalization using controlled antegrade and retrograde subintimal tracking: the CART technique. J Invas Cardiol 2006; 18: 334-8.

[19] Tsuchikane E, Katoh O, Kimura M, et al. The first clinical experience with a novel catheter for collateral channel tracking in retrograde approach for chronic coronary total occlusions. J Am Coll Cardiol Interv 2010; 3 : 165-71.

[20] Nguyen TN, Hu D, Chen SL, editors. Practical Handbook of Advanced Interventional Cardiology. $4^{\text {th }}$ ed. Wily-Blackwell; 2013.

[21] Cohen R, Hattab M, Elhadad S. Retrograde approach "reverse CART technique" with a single guide for chronic total occlusion of the right coronary via an anomalous left circumflex artery. J Invas Cardiol 2011; 23: E92-94.

[22] Rathore S, Katoh O, Tsuchikane E, et al. Mini-Focus Issue: Chronic total occlusion a novel modification of the retrograde approach for the recanalization of chronic total occlusion of the coronary arteries intravascular ultrasound-guided reverse controlled antegrade and retrograde tracking. J Am Coll Cardiol Interv 2010; 3: 155-64.

[23] Funatsu A, Kobayashi T, Nakamura S. Use of kissing microcatheter technique to exchange a reytrograde wire. J Invas Cardiol 2010; 22: E74-7.

[24] Ge JB, Zhang F, Ge L, et al. Wire trapping technique combined with retrograde approach for recanalization of chronic total occlusion. Chin Med J 2008; 121: 1753-6.

[25] Ge JB, Zhang F. Retrograde recanalization of chronic total coronary artery occlusion using a novel "reverse wire trapping" technique. Cathet Cardiovasc Interv 2009; 74: 855-60.

[26] Kim HM, Yu LH, Mitsudo K. A new retrograde wiring technique technique for chronic total occlusion. Cathet Cardiovasc Interv 2010; 75: 117-9.

[27] Wu E, Chan W, Yu CM. The confluent balloon technique-two cases illustrating a novel method to achieve rapid wire crossing of chronic total occlusion. J Invas Cardiol 2009; 108: 548-53.

[28] Grantham JA. The steps of the retrograde technique: wire externalization, stenting and wire removal. Intervent Cardiol Clin 2012; 1: 345-48.

[29] Hashidomi H, Saito S. Dilatation of septal collateral artery and subsequent cardiac tamponade during retrograde percutaneous coronary intervention using a microcatheter for chronic total occlusion. J Invas Cardiol 2011; 24: 74-6.

[30] Nombela-Franco L, Werner GS. Retrograde recanalization of a chronic total occlusion of the left anterior descending artery. J Invas Cardiol 2010; 22: E7-12. 